\title{
Adnectin CT-322 inhibits tumor growth and affects microvascular architecture and function in Colo205 tumor xenografts
}

\author{
MAXIMILIAN ACKERMANN ${ }^{1}$, IRVITH M. CARVAJAL ${ }^{2}$, BRENT A. MORSE ${ }^{2}$, MIGUEL MORETA ${ }^{2}$, \\ STEVEN O'NEIL ${ }^{2}$, SYLVIE KOSSODO ${ }^{3}$, JEFFREY D. PETERSON ${ }^{3}$, VERA DELVENTHAL ${ }^{1}$, \\ H. NICHOLAS MARSH ${ }^{2}$, ERIC S. FURFINE ${ }^{2}$ and MORITZ A. KONERDING ${ }^{1}$ \\ ${ }^{1}$ Institute of Functional \& Clinical Anatomy, University Medical Center of the Johannes Gutenberg University Mainz, \\ Mainz, Germany; ${ }^{2}$ Adnexus, a Bristol-Myers Squibb R\&D Company, \\ Waltham, MA; ${ }^{3}$ VisEn Medical Inc., Bedford, MA, USA
}

Received August 20, 2010; Accepted October 19, 2010

DOI: 10.3892/ijo_00000825

\begin{abstract}
Antiangiogenesis has become a promising pillar in modern cancer therapy. This study investigates the antiangiogenic effects of the PEGylated Adnectin ${ }^{\mathrm{TM}}, \mathrm{CT}-322$, in a murine Colo-205 xenograft tumor model. CT-322 specifically binds to and blocks vascular endothelial growth factor receptor (VEGFR-2). Adnectins are a novel class of targeted biologics engineered from the 10th domain of human fibronectin. CT-322 treated tumors exhibited a significant reduction in tumor growth of $69 \%$, a 2.8 times lower tumor surface area and fewer necrotic areas. Control tumors showed a 2.36-fold higher microvessel density (MVD) and a 2.42 times higher vessel volume in corrosion casts. The vascular architecture in CT-322-treated tumors was characterized by a strong normalization of vasculature. This was quantified in corrosion casts of CT-322 treated tumors in which the intervascular distance (a reciprocal parameter indicative of vessel density) and the distance between two consecutive branchings were assessed, with these distances being 2.21 times and 2.37 times greater than in controls, respectively. Fluorescence molecular tomography (FMT) equally affirmed the inhibitory effects of CT-322 on tumor vasculature as indicated by a $60 \%$ reduction of the vascular probe, AngioSense, accumulating in tumor tissue, as a measurement of vascular permeability. Moreover, AngioSense accumulation was reduced as early as $24 \mathrm{~h}$ after starting treatment. The sum of these effects on tumor vascu-
\end{abstract}

Correspondence to: Dr Moritz A. Konerding, Institute of Functional \& Clinical Anatomy, University Medical Center of the Johannes Gutenberg-University Mainz, D-55099 Mainz, Germany

E-mail: konerdin@uni-mainz.de

Abbreviations: VEGF, vascular endothelial growth factor; FMT, fluorescence molecular tomography

Key words: Adnectin, antiangiogenesis, tumor microvasculature, corrosion casting, fluorescence molecular tomography, xenograft lature illustrates the anti-angiogenic mechanism underlying the antitumor activity of CT-322 and provides support for further evaluation of this Adnectin in combinatorial strategies with standard of care therapies.

\section{Introduction}

Angiogenesis is one of the growth-influencing steps for numerous pathologic processes, in particular for tumor growth (1). Tumorigenesis and tumor progression depend on an 'angiogenic switch' whereby the tumor initiates recruitment of its own blood supply, a process which occurs at different stages of tumor progression pathways, depending on the tumor type and tumor microenvironment (2).

Tumor blood vessels differ both in architecture and structure from their normal physiological counterparts. Tumor vessel systems are characterized by a lack of normal hierarchy, poor vessel wall construction without medial layers, missing differentiation into structurally established arteries and veins, tortuous courses with dead ends, and irregular, dilated sinusoidal vessel diameters $(3,4)$. These differences in morphological features of tumor vasculature may be related to a deficiency in pericyte function and an abnormal expression of angiogenic molecules $(5,6)$.

The importance of the inhibition of angiogenesis as a component of therapeutic oncology concepts is increasing. Antiangiogenic agents may be used not only for the treatment, but also for the prevention of tumor recurrence or metastasis (7). Currently, numerous VEGF pathways blocker groups such as antibodies, small molecules, siRNAs and traps are in clinical development or already commercially available. They show various different target patterns. Unfortunately, toxicity [e.g., hypertension and thrombotic events (8)] and selectivity are the limiting factors in several antiangiogenic approaches.

It has been proposed that the blockade of angiogenic pathways could result in a paradoxical remodeling of tumor vasculature. This phenomenon is associated with a selective transient pruning of poorly formed vessels and leads to a temporary improvement of blood flow and oxygen delivery (9). This vessel remodeling approach using antiangiogenic 
drugs has been associated with the improvement of the efficacy of chemotherapy in a combinatory therapy approach $(10,11)$.

Thus, inhibition of angiogenic pathways may result both in tumor regression and in a normalized vascular bed which can result in improved delivery of chemotherapy and a corresponding increase in efficacy. These two aspects play, besides toxicity, a pivotal role in multidrug chemoantiangiogenic therapy (12).

Adnectins are a new class of biologics engineered from the tenth type III domain of human fibronectin. They represent a promising alternative to antibodies and small molecules due to their stable pharmacological properties and their selectivity (13). Adnectins are pharmaceutically highly-suited to their therapeutic disease target and can be designed for receptors, ligands or proteins with a high nanomolar or picomolar affinity, potency and specificity by varying the amino acid sequence of three clustered targeting loops (14). The PEGylated Adnectin CT-322 binds and blocks the activity of VEGFR-2, a key player in tumor angiogenesis, resulting in reduced endothelial cell function, as determined preclinically in vitro and in vivo (15-18). This therapeutic approach differs from that of other antiangiogenic drugs (e.g., sorafenib and sunitinib) in terms of its selectivity on this specific VEGFR2pathway $(17,18)$.

The aim of this study was to elucidate possible structural, architectural and functional changes in the tumor microvasculature by treatment with CT-322 and its impact on tumor growth.

\section{Materials and methods}

Animals and compounds. All experiments were conducted with 6-8-week-old female athymic NCRNU-M-F nude mice (Taconic, Hudson, NY) housed in an SPF facility on a 12-h light/dark cycle, with food and water available ad libitum. The Adnectin compound CT-322 and vehicle were provided by Adnexus, a Bristol-Myers Squibb R\&D Company, Waltham, MA. All experiments had previously been approved by the regional animal ethics committee and were conducted in accordance with the relevant laws for animal protection.

Colo205 xenograft model and treatment. The human colorectal carcinoma line Colo205 (ATCC-CCL222) was obtained from the American Type Culture Collection (ATCC; Manassas, VA). Cells were grown and maintained according to ATCC instructions. Subconfluent cultures were harvested, washed and resuspended in PBS. Colo205 xenografts were inoculated by subcutaneous injection of $5 \times 10^{6}$ Colo205 cells. Tumors were measured three times per week using a caliper and the tumor volume was calculated using the formula $\pi / 6 \mathrm{x}$ (larger diameter) $\mathrm{x}$ (smaller diameter) ${ }^{2}$. Based on previous experiments, the determined optimum dose of CT-322 of $60 \mathrm{mg} / \mathrm{kg}$ body weight (18) three times per week was used on for the experiments described here. Control animals received an equivalent volume of the drug vehicle $(100 \mu 1,5 \mathrm{ml} / \mathrm{kg}$ body weight). The first treatment started seven days after tumor cell inoculation, when the mean tumor volume was $\sim 150 \mathrm{~mm}^{3}$. The animals were sacrificed after three weeks of treatment. Tumors from both groups were collected and processed for histology and vascular corrosion casting.
Vascular corrosion casting. Briefly, at the time of tumor collection, heparin (500 IU) was administered to the mice. Mice were thoracotomized in deep anesthesia with pentobarbital (Sigma-Aldrich, Munich, Germany). The ascending aorta was cannulated with an olive-tipped cannula via the left ventricle. The vasculature was flushed with saline (at body temperature) followed by glutaraldehyde fixation solution (2.5\%, pH 7.4, Sigma-Aldrich). Fixation was followed by injection of a prepolymerized methylmetacrylate (Mercox, Japan Vilene Co., Tokyo, Japan) diluted with $20 \%$ methylmethacrylate monomers as casting medium. After curing of the resin, the casted mice were stored at $-20^{\circ} \mathrm{C}$ until further processing. The tumors as well as the kidneys were dissected and macerated in $10 \% \mathrm{KOH}$ (Fluka, Neu-Ulm, Germany) at $40^{\circ} \mathrm{C}$ over 2-3 days. Specimens were then rinsed with water and frozen in distilled water. The casts were freeze-dried, cut and mounted on specimen holders, sputtered with gold in an argon atmosphere and examined using a Philips ESEM XL-30 scanning electron microscope (Philips, Eindhoven, The Netherlands).

$3 D$ morphometry. For quantitative analyses, stereopairs with a tilt angle of $6^{\circ} \mathrm{C}$ were collected from each tumor using a eucentric specimen holder. The stereopairs were used for morphometry of parameters defining the architecture of the microvascular unit, namely the intervascular distances, the interbranching distances (vessel segment lengths) and vessel diameters. For this, the stereo pairs were color coded and reconstructed as anaglyphic images. With the known tilt angle, calculations of individual points marked interactively in both images of each stereopair were carried out using macros defined for the KS 300 software (Kontron Electronics, Eching, Germany).

Tissue sampling and histology. Six tumors from both the control and test groups underwent histology, immunohistochemistry and ultrastructural studies. Half of each tumor was processed after fixation by immersion for conventional H\&E staining. Using a Leica MS 5 stativ (Leica Microsystems, Germany) equipped with a JVC KY-F75U C mount digital camera (JVC, Yokohama, Japan), all sections were scanned on a light panel LP-200 cold cathode light panel (Universal Electronics Industries) and stored and analyzed using the Diskus morphometry software (Diskus 4.50, Hilgers, Königswinter, Germany).

Microvessel densities were determined in anti-CD31 stained frozen sections. Immunohistochemical staining of endothelial cells was performed using a monoclonal antibody against CD31 (BD Biosciences Pharmingen, Heidelberg, Germany). Antibody binding was visualized via a three-step staining procedure using a biotinylated polyclonal anti-rat IgG secondary antibody (DakoCytomation GmbH, Hamburg, Germany) and the streptavidin horse-radish peroxidase reaction together with the DAB detection system (DakoCytomation). The vessel densities were assessed in coronary sections of the tumors using a Weibel grid (19) and expressed as percentual vessel surface area.

Tumor samples designated for transmission electron microscopy were fixed with $2.5 \%$ buffered glutaraldehyde and processed according to standard protocols. After embedding 
in Epon (Serva, Heidelberg, Germany), 700 Ả ultrathin sections were analyzed using a Leo 906 digital transmission electron microscope (Leo, Oberkochen, Germany).

Fluorescence molecular tomography (FMT). Longitudinal in vivo functional mapping of the antiangiogenic and antitumorigenic effects of CT-322 was done in mice with established subcutaneous Colo205 tumors that were randomized into vehicle and CT-322 groups (12 mice each) of equivalent average starting tumor volume $\left(\sim 325 \mathrm{~mm}^{3}\right.$ per group). Tumor volume was evaluated as described earlier in this section, focusing on time-points coinciding with agent imaging. Imaging was conducted using FMT (FMT-Solaris, VisEn Medical, Bedford, MA) with AngioSense ${ }^{\mathrm{TM}} 680$ (VisEn Medical, Bedford, MA) as the vascular molecular probe. AngioSense is constitutively fluorescent and has a MW of $250 \mathrm{kDa}$ that allows monitoring of vascular leakage. It has a half-life in plasma of $6 \mathrm{~h}$, is exclusively intravascular $1 \mathrm{~h}$ after injection and extravasates at sites of increased vascular permeability by $24 \mathrm{~h}$, allowing the detection of blood pooling in tumors. Mice were injected intravenously with 2 nmoles of AngioSense 680 immediately after intraperitoneal administration of treatments. FMT imaging was performed $24 \mathrm{hr}$ post- probe injection on days $1,7,14$ and 21 , in order to determine the time frame for maximal vascular effects. FMT was carried out using the VisEn FMT1 system.

NIR imaging. On the day of imaging, mice were anesthetized by i.p. injection of Ketamine $(100 \mathrm{mg} / \mathrm{kg})$ and Xylazine $(20 \mathrm{mg} / \mathrm{kg})$. Anesthetized mice were positioned in the FMT imaging chamber and sequential reflectance and tomographic datasets were generated using near-infrared (NIR) laser light. Briefly, for optical tomographic imaging a low power NIR laser diode transilluminated (i.e., passed light through the body of the animal to be collected on the opposite side) the lower torso region by scanning through numerous sites in and around the tumor. Signal detection was via a thermoelectrically cooled CCD camera placed on the opposite side of the imaged animal to collect the multiple transillumination images. Appropriate optical filters allowed collection of both fluorescence and excitation datasets, and the multiple sourcedetector fluorescence projections were normalized to the paired collection of laser excitation data.

The collected fluorescence data were reconstructed by FMT system software as three-dimensional (3D) fluorescence datasets showing signal within the flank tumors. To quantify this tumor fluorescence, 3D regions of interest (ROI) were drawn encompassing the tumors, and a threshold was applied identically to all animals that was equal to $20 \%$ of the mean tumor fluorescence $(12 \mathrm{nM})$ for the vehicle-treated tumor group. This minimized the quantification of extra-tumoral low-intensity, background fluorescence but allowed optimal quantification of high fluorescent signal in areas of vascular leak of AngioSense. The total amount of tumor fluorescence (in pmoles) was automatically calculated relative to internal standards generated with known concentrations of appropriate NIR dyes. Because vascular agents can potentially generate high background tissue signal, the non-tumor associated fluorescence was assessed in adjacent background flank areas within each mouse scan. For visualization purposes, FMT

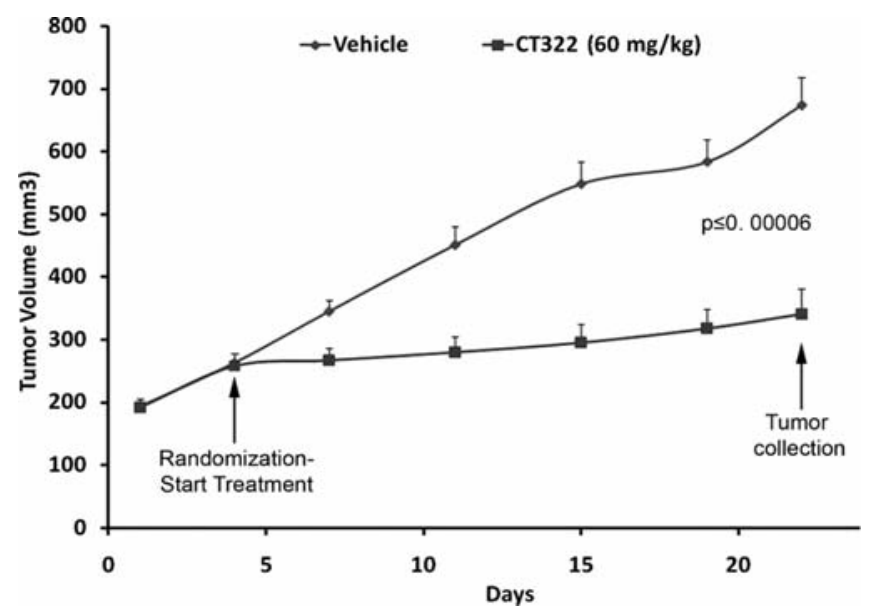

Figure 1. Growth curves of Colo205 tumors on nude mice treated with vehicle $(n=14)$ or CT-322 $(n=14)$. CT-322 was dosed three times a week (i.p.) with $60 \mathrm{mg} / \mathrm{kg}$ body weight. Treatment started seven days after inoculation. Tumors were collected for histology and microvascular corrosion casting at day 22 .

system software provided maximum intensity projection images of the 3D fluorescence in the tumors into a $2 \mathrm{D}$ representation. FMT imaging was routinely performed prior to each tomographic imaging session using built-in LED front illuminators and collection of single camera images.

Influence of CT-322 on normal tissue. In order to elucidate the effects of CT-322 on the vessel architecture of normal tissue, a histopathological reading of semithin and ultrathin sections of kidneys was performed. Additionally, corrosion casts (methodology see above) of the kidney vessel architecture were assessed.

Statistical analysis. For all comparisons, the unpaired Student's t-test for samples of unequal variances was used to calculate statistical significance. Data are expressed as mean \pm SD. The significance level for the sample distribution was defined as $\mathrm{p} \leq 0.05$.

\section{Results}

Tumor growth and mean survival time. CT-322 was seen to effectively suppress growth of established Colo205 colon carcinoma xenografts with a dose of $60 \mathrm{mg} / \mathrm{kg}$ administered intraperitoneally, three times weekly (TIW), by $69 \%$ after tumors had attained a mean volume of $150 \mathrm{~mm}^{3}(\mathrm{p} \leq 0.00006$, Fig. 1). With this dosage a $100 \%$ survival was achieved.

Histopathology. All specimens (whether from control or CT-322 treated animals) consisted of mainly solid infiltrates of an undifferentiated, mitotically active adenocarcinoma with some glandular differentiation and focal necroses, which were partially confluent and reached, in places, up to the tumor capsule (Fig. 2A). Typically, islands of vital tumor tissue were seen within necrotic areas. In the center of these islands a major sinusoidal vessel was frequently seen (Fig. 2B). Semithin sections showed that these vessels within vital areas were, in most cases, functionally open and not occluded. 

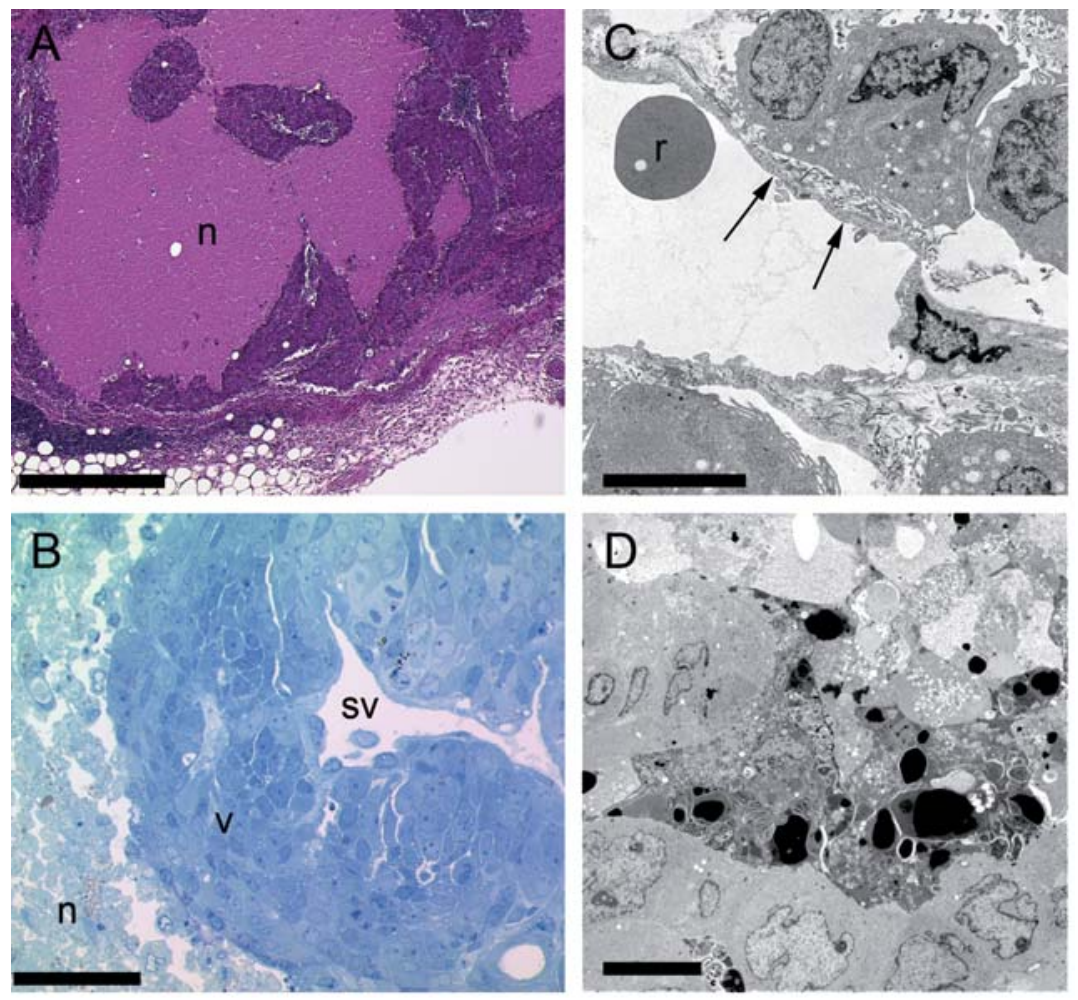

Figure 2. (A) H\&E stained section of untreated Colo205 tumor with necrotic areas (n) reaching to the capsule infiltrating the s.c. fatty tissue. (B) Viable tumor tissue island (v) in necrotic area (n) with lacunary, sinusoidal vessel (sv). Semithin section, toluidine blue. (C) Transmission electron microscopy of untreated tumor vessel with low structural stability. r, red blood cell; arrows, endothelium. (D) Apoptotic bodies in hypoxic areas. Bars in: (A), $200 \mu \mathrm{m} ;$ (B) $50 \mu \mathrm{m}$; (C) $5 \mu \mathrm{m}$; and (D) $3 \mu \mathrm{m}$.

A

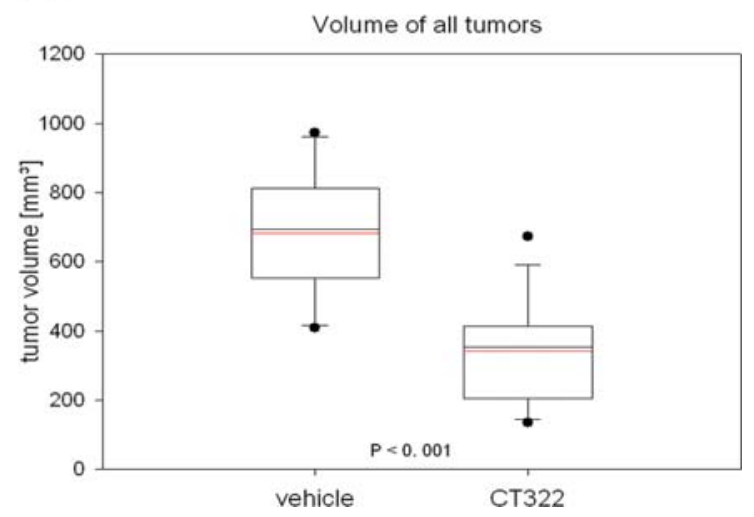

C

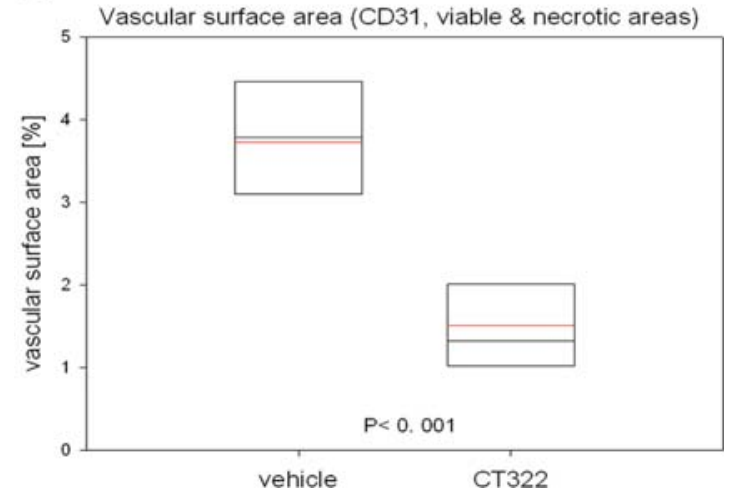

B

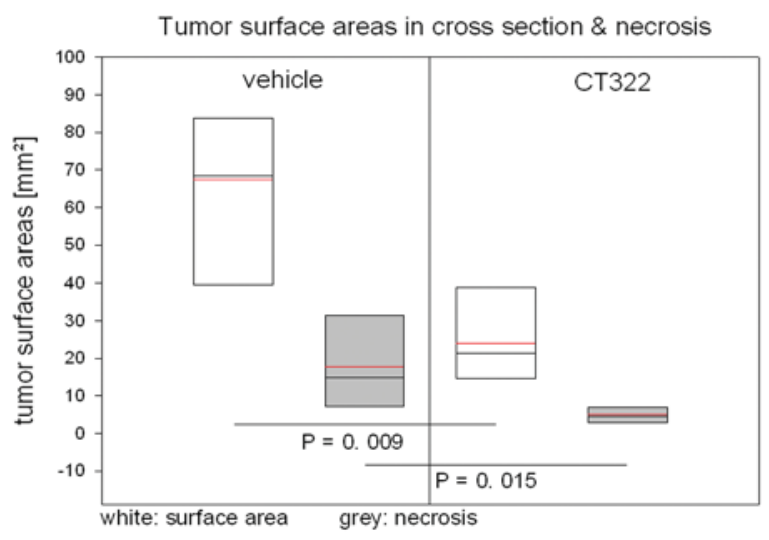

D

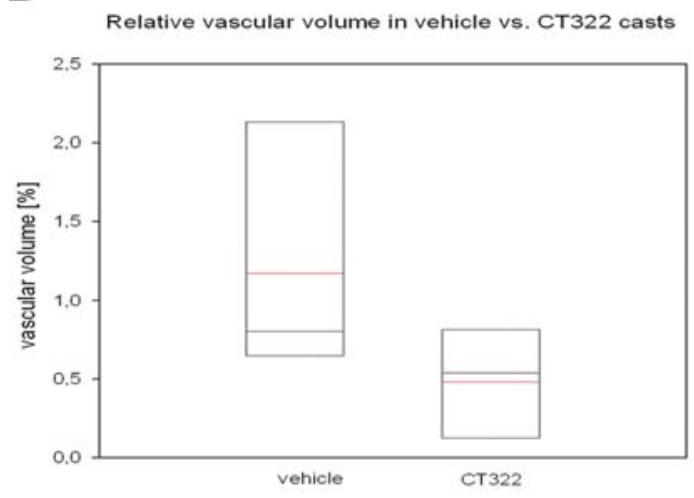

Figure 3. (A) Tumor volumes at 22 days under treatment. (B) Tumor and necrosis surface areas in cross-sections. (C) Percentual vascular surface area in CD31 stains. (D) Percentual relative vascular volume determined in corrosion casts; mean (red line), median (black line). 

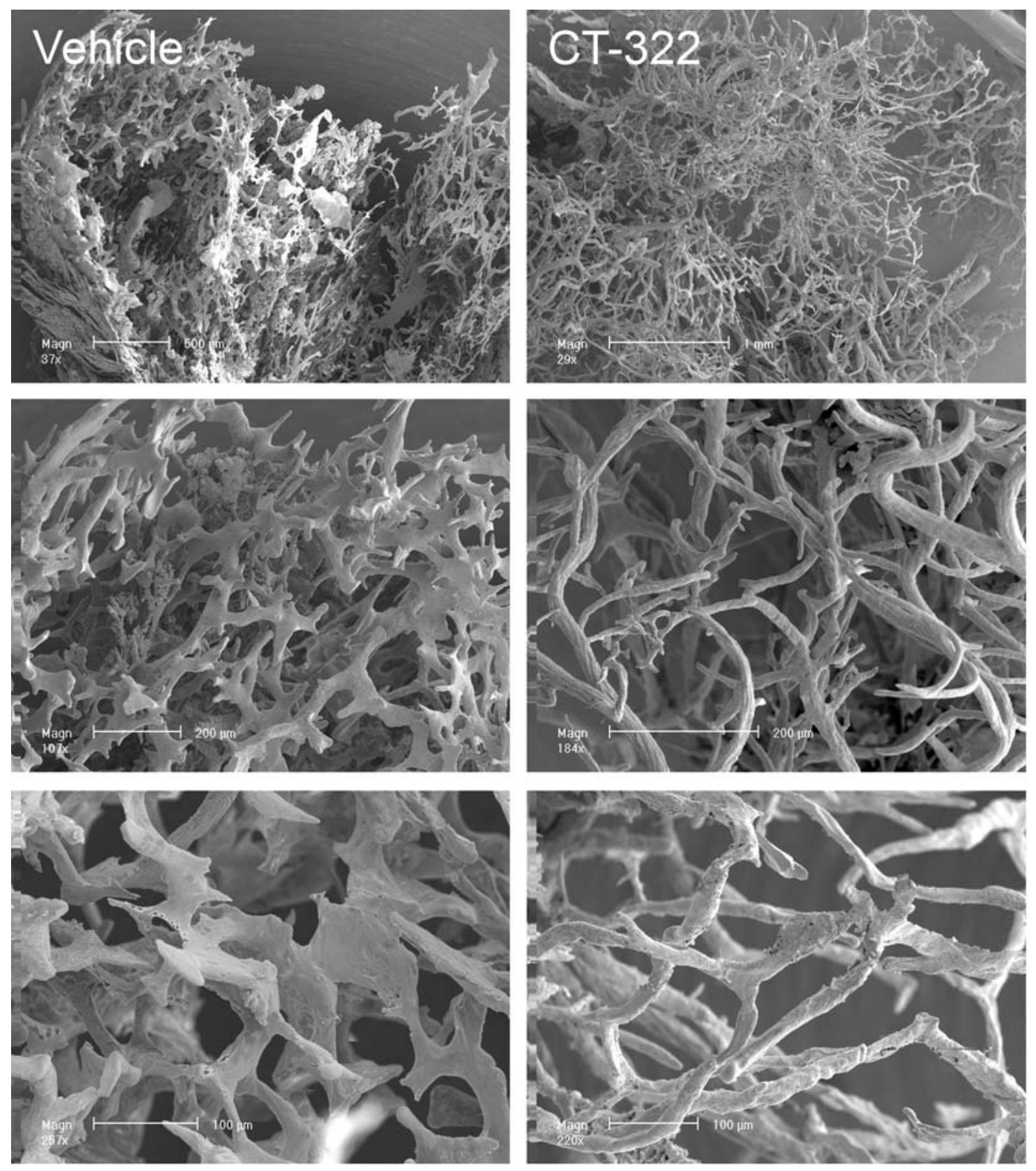

Figure 4. Scanning electron microscopic images of vascular corrosion casts of control tumors (left panel) and CT-322-treated tumors (right panel). Treatment with CT-322 Adnectin was associated with a normalization of tumor vessel architecture. Note the higher heterogeneity of vessel densities and vessel diameters in the vehicle treated tumors.

The vessel diameters appeared irregular, the endothelium was in nearly all cases flatly extended and partially missing. Differentiated arteries and veins were seen only in close proximity to incorporated connective tissue stalks. The vast majority of vessels showed a sinusoidal vessel wall construction with low structural stability (Fig. 2C). In places there were sprouts with higher endothelium, whereby even sprouts or juvenile forms frequently showed degenerative signs in the form of hypoxia-induced vacuolization. Apoptotic bodies indicative of programmed cell death were seen in both vessel wall confining cells and tumor cells (Fig. 2D). No structural differences in the vascularity were observed between vehicle- and CT-322 treated tumors.

Tumor volumes and tissue viability. Fig. 3A shows that the mean volume of the CT-322 treated tumors $\left(341.4 \pm 148.2 \mathrm{~mm}^{3}\right)$ were significantly smaller than that of the control tumors $\left(674.4 \pm 164.2 \mathrm{~mm}^{3}\right) . \mathrm{H} \& \mathrm{E}$ stained coronary sections showed reduced surface areas $\left(23.9 \pm 12.5 \mathrm{~mm}^{2}\right.$ vs. $67.5 \pm 27.3 \mathrm{~mm}^{2}$ for treated and untreated tumors, respectively) as well as reduced amounts of necroses in the treatment group $\left(5.0 \pm 3.3 \mathrm{~mm}^{2} \mathrm{vs}\right.$. $17.8 \pm 10.6 \mathrm{~mm}^{2}$; Fig. 3B).

Vessel density and relative vascular volume. The percentual vascular surface areas as assessed in low power magnification anti-CD31 stains are shown in Fig. 3C. Treated tumors had a mean vessel area of $1.57 \pm 0.58 \%$, compared to $3.70 \pm 0.81 \%$ in the controls $(p<0.0001)$. This results in a ratio of $1: 2.36$. This result is comparable to the ratios of the relative vascular volumes assessed in corrosion casts by weighing tumors before and after maceration, which was 1:2.42. The results in Fig. 3D are expressed as a percentage of vessel volume (vessel replica weight after maceration) of the total weight: the vessel volume fraction for the CT-322 treated animals was $0.48 \pm 0.35 \%$, whereas the controls have were seen to have a significantly higher relative vascular volume of $1.17 \pm 0.76 \%(\mathrm{p}<0.043)$. 
A
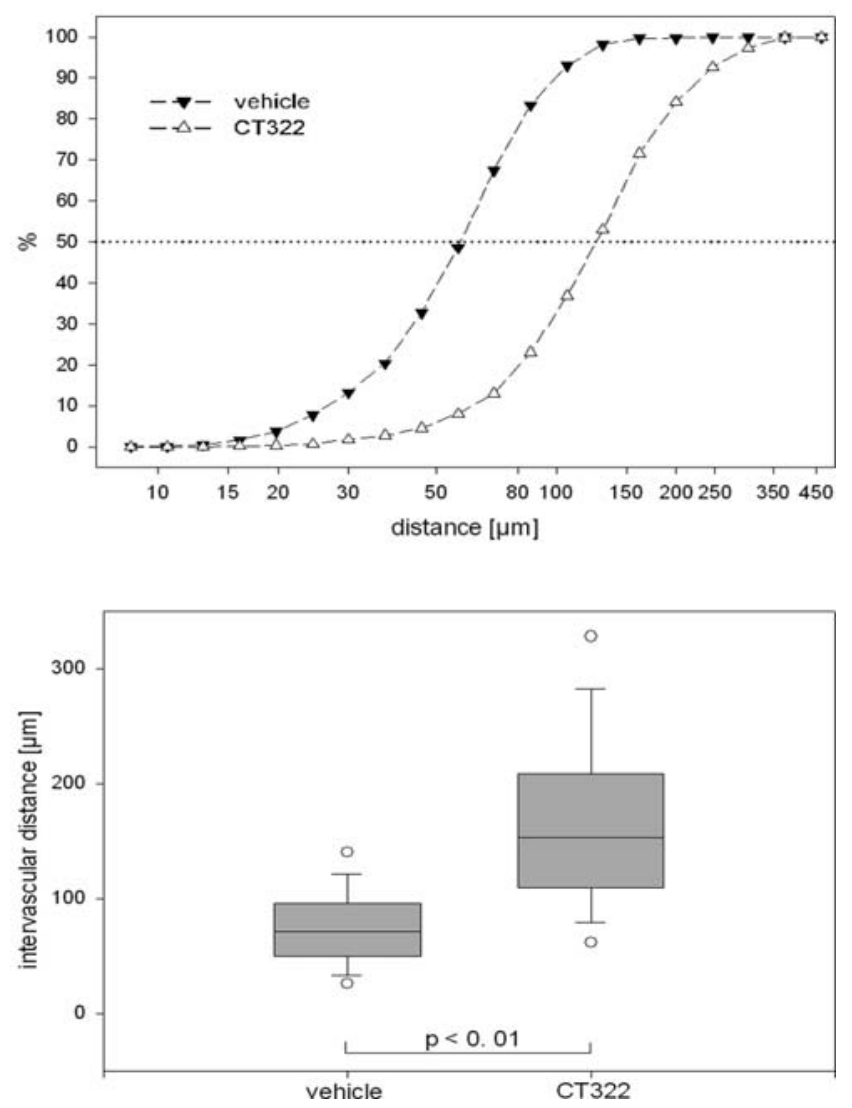

C
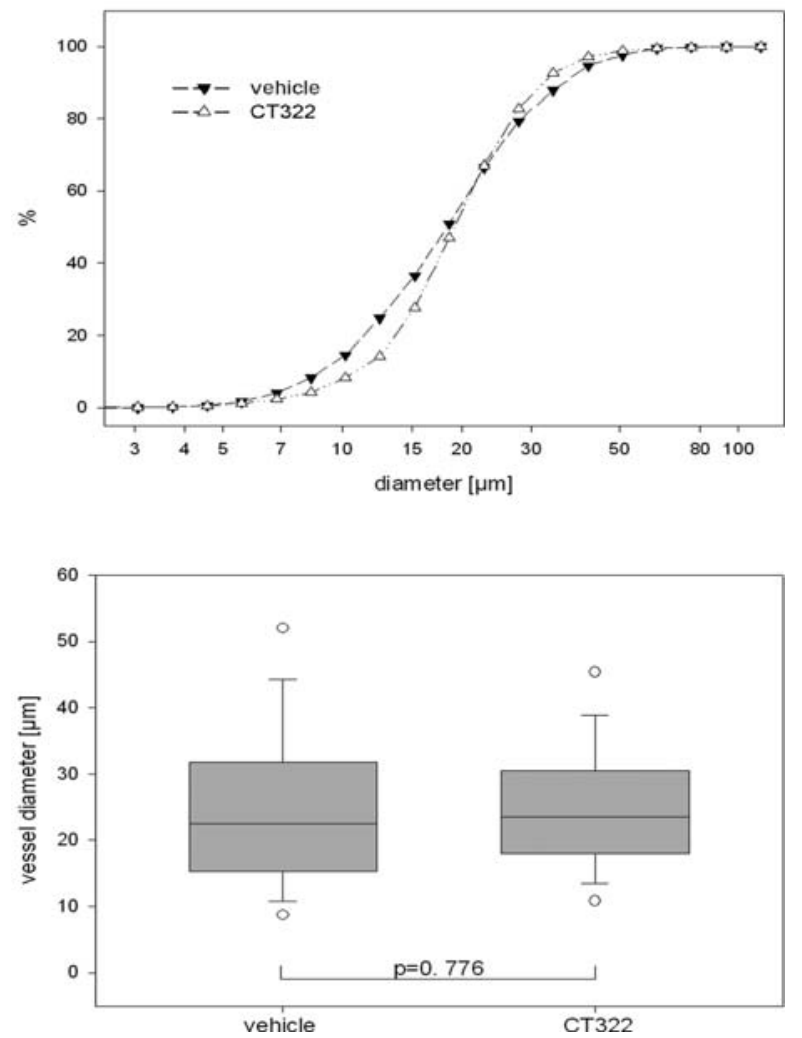

Figure 5. Architectural parameters describing the tumor microvascular unit: intervascular distances (A), interbranching distances (B) and vessel diameters $(\mathrm{C})$, expressed as cumulative percentual frequency graphs (top) and box plots of absolute (bottom). Boxes indicate 25th, 50th, 75th percentile, whiskers 10 th and 90 th, dots 5 th and 95 th percentile.
B
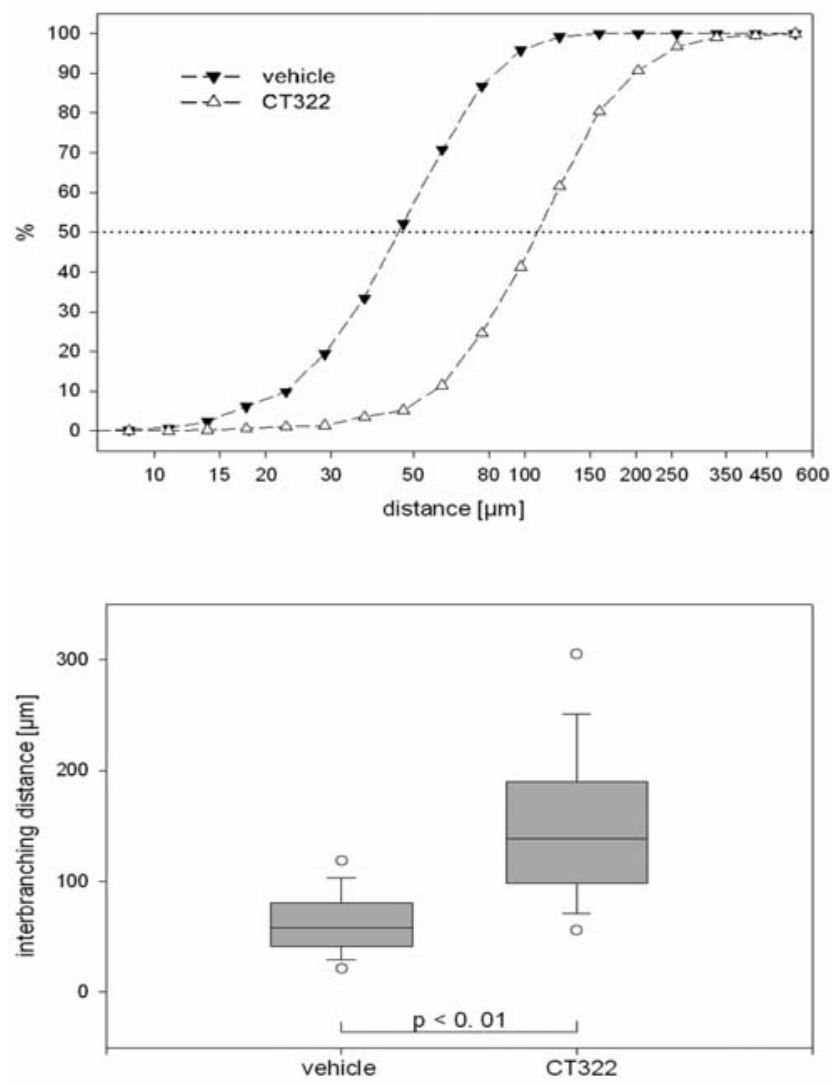

When comparing the almost identical ratios in the vessel surface area and the relative vascular volume, it should be kept in mind that the former results were obtained by counting all vessels in a $2 \mathrm{D}$ section, whereas the latter represents only the luminal volumes of the functionally open vessels at the time of perfusion.

Microvascular architecture. The vehicle-treated tumors were so qualitatively different from the CT-322 treated ones in terms of vessel architecture that an independent examiner was able to correctly assign all blinded tumor casts to the correct groups. The vehicle-treated tumors displayed a chaotic arrangement with less hierarchy and higher vascular densities (Fig. 4, left panel).

In CT-322 treated tumors (Fig. 4, right panel) the microvascular architecture more closely resembled by far the normal, autochthonous subcutaneous vascular architecture. The diameters of the individual vessel segments showed only little variations, whereas the controls were characterized by large caliber, sinusoidal vessel networks with frequent vessel diameter changes.

Tumors of both groups induced a peritumoral, desmoplastic 'vascular envelope' as frequently seen in xenografts. In general, this vascular envelope is more densely expressed in the controls than in the tests treated with CT-322, however, the average size of the controls was significantly higher and, thus, the stimulus for vessel induction was more pronounced.

$3 D$ quantitative data on microvascularity. The results of parameters defining the microvascular unit are depicted in 
A

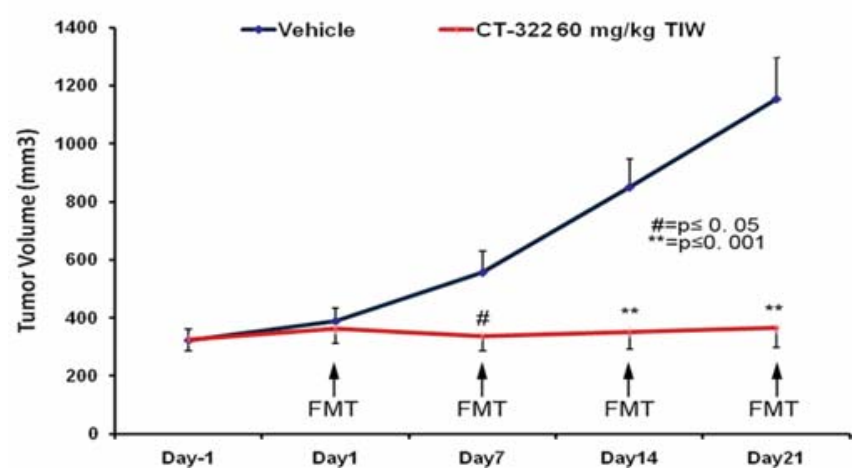

B

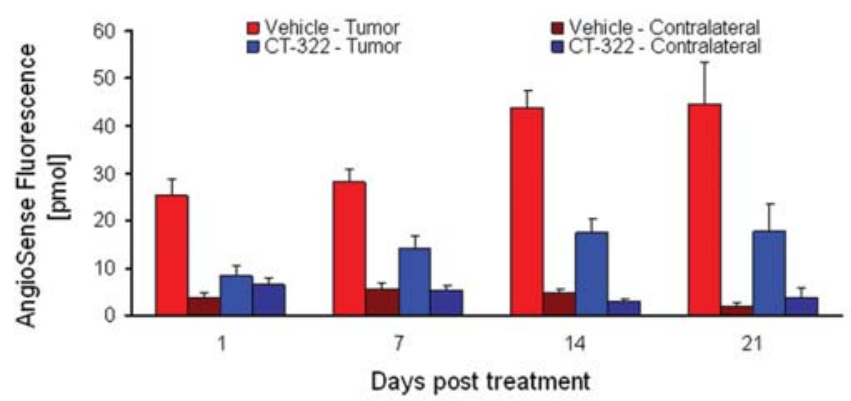

C

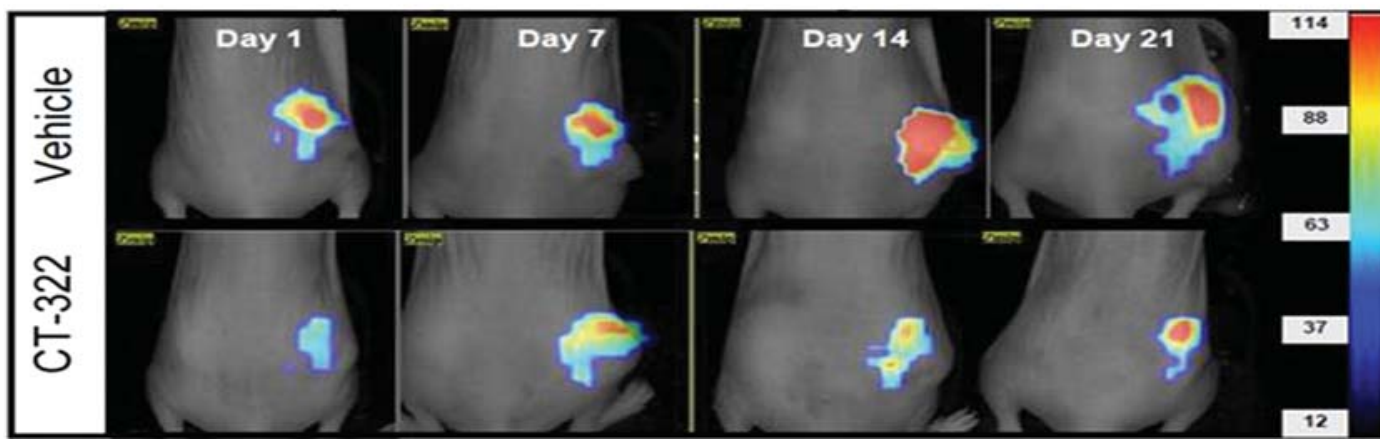

Figure 6. Fluorescence molecular tomography. (A) Growth curves of Colo205 tumors on nude mice treated with vehicle (n=12) or CT-322 (n=12). (B) Tumor Vascularity, AngioSense fluorescence quantitation (pmol) on days 1, 7, 14 and 21. Note the increase in fluorescence on days 7-21 in the vehicles that is paralleled by increases in tumor size. Non-tumor associated fluorescence was assessed in adjacent background flank areas within each mouse scan (contralateral). (C) Tumor Vascularity, AngioSense fluorescence images acquired $24 \mathrm{~h}$ post AngioSense injection. Representative controls vs. CT-322 treated mice.

Fig. 5 in the form of both cumulative frequency graphs and box plots. The mean intervascular distance (distance between two adjacent vessels) is with $169.1 \mu \mathrm{m}$ in treated tumors $2.21 \mathrm{x}$ larger than in the more densely vascularized untreated controls which exhibited a mean intervascular distance of $76.5 \mu \mathrm{m}$.

The parameter interbranching distance refers to the individual vessel's segment length. The more frequently a vessel produces new branches, the shorter is the interbranching distance. In this study, the untreated control tumors had a mean vessel segment length (interbranching distance) of $64.4 \mu \mathrm{m}$, whereas the CT-322 treated tumors' interbranching length was $152.9 \mu \mathrm{m}$ and thus 2.43 times longer (Fig. 5B). On the contrary, the mean vessel diameters, did not show significant differences (Fig. 5C) although there was a tendency towards more uniform vessel diameters in the CT-322 treated tumors.

Fluorescence molecular tomography. Progressive growth of Colo205 tumors, as assessed by calculated tumor volume, was evident in control animals, and was significantly reduced by CT-322 treatment by day 7 post-treatment, continuing until day 21 ( $\mathrm{p} \leq 0.001$, Fig. 6A). The assessment of tumor masses using the fluorescencent vascular probe, AngioSense, qualitatively and quantitatively corroborated the calculated volume measurements of tumor growth (Fig. 6B and C).

FMT revealed, already one day after the first treatment, strikingly lower AngioSense fluorescencies in CT-322 treated tumors than in controls $(8.4 \pm 6.5$ pmol vs. $25.4 \pm 10.7$ pmol, respectively; $\mathrm{p}=0.0013)$. These differences were evident in the absence of an effect on tumor size (Fig. 6A). This decrease in AngioSense fluorescence continued throughout the duration of the study and was statistically significant at all time-points (Fig. 6C).

The average background fluorescence (contralateral flanks, $\sim 3.4$ pmols) did not decrease over time (black line in Fig. 6C). The lower detection of AngioSense fluorescence in the CT-322 treated tumors compared to vehicle treated tumors over the treatment period confirms the smaller vascular volumes seen in corrosion casts and further suggests a functional change in vascular permeability and/or leakiness compared to untreated tumors. The 2-fold increase in AngioSense fluorescence between days 1 and 21 in the CT-322 treated tumors (Fig. 6C) in the absence of overall changes in tumor size over that time may indicate either a remodeling of the vascular spaces or may reflect a gradual increase in vascular permeability compared to earlier time-points. FMT quantified a 1:2.5 ratio of fluorescence in CT-322 treated tumors versus controls on day 21 after treatment, a value comparable to that obtained for the vascular volumes by means of microvascular corrosion casting.

Influence of CT-322 on kidney vasculature. Neither transmission electron microscopy of ultrathin sections nor histology or microvascular corrosion casting revealed any changes in kidney microvasculature between controls and tests (data not shown). 


\section{Discussion}

Adnectins are a new class of targeted biologics based on engineered amino acid variants of the 10th human fibronectin type III domain $(10 \mathrm{Fn} 3)(13,14)$. CT-322 is a PEGylated Adnectin designed to specifically bind and block human VEGFR-2 $\left(\mathrm{K}_{\mathrm{D}}, 11 \mathrm{nM}\right)$ with no detectable cross-reactivity to human VEGFR-1 and VEGFR-3 (15-18). The initial bioengineering process of CT-322 was designed to retain substantial affinity to the mouse VEGFR-2 $\left(\mathrm{K}_{\mathrm{D}}, 250 \mathrm{nM}\right)(18)$. This biochemical property has made possible the evaluation of anti-tumor and antiangiogenic activities of CT-322 in tumor xenograft mouse models. CT-322 and its blocking activity against mouse VEGFR-2 resulted in an effective inhibition of tumor growth when administered at doses of up to $60 \mathrm{mg} / \mathrm{kg}$ in a human Colo205 xenograft model in nude mice (18).

CT-322 also significantly inhibited tumor growth of the U87 glioblastoma, and lung metastasis in an orthotopic MDAMB-231 breast carcinoma model, with equivalent activities to that of anti-VEGFR-2 antibody (DC101) and anti-VEGF-A antibody (bevacizumab), respectively (18). Specific suppression of VEGFR-2 signaling may have both efficacy and safety advantages compared to either specific VEGF-A blockade or inhibition of multiple tyrosine kinases by small molecules. VEGF-A signals through both VEGFR-1 and R-2, though signaling through VEGFR-2 is considered to be the primary driver of angiogenesis (20). Furthermore, while VEGF-C and VEGF-D are often considered activators of lymphangiogenesis (21), they also signal through VEGFR-2 and are up-regulated in many tumors $(22,23)$. Specific blockade of VEGF-A (e.g., bevacizumab) blocks some signaling through VEGFR-1 and VEGFR-2 but does not block signaling of VEGF-C or -D through VEGFR-2. Therefore, additional efficacy may be gained from VEGFR-2 blockade by blocking angiogenic signaling from VEGF-C and -D. A potential safety advantage may be gained from not blocking VEGFR-1 (or other tyrosine kinases) which might result in undesired side effects.

The studies described here demonstrate that effective suppression of tumor growth by CT-322 was associated with a potent anti-angiogenic effect on tumor vasculature, characterized by a reduction in the total tumor vasculature, intervascular distance and interbranching distance, as assessed using scanning electron micrographs of tumor vascular casts. Functional repetitive molecular tomographic studies showed almost identical results to those obtained in morphologic and morphometric studies (24). These anti-tumor and anti-vascular effects are similar to those seen with other anti-angiogenic VEGF pathway-blocking therapies $(25,26)$.

The intervascular distances measured using 3D morphometry in the present study showed an increase of $121 \%$, and of the interbranching distances of $143 \%$. When comparing the efficacy of the compound in terms of vessel reduction and changes in microvascular architecture with other agents, a better efficacy with CT-322 was seen to be obtained than with any other compound. PTK787/ZK 222584 for instance, a specific VEGF-receptor tyrosine kinase inhibitor, also affected the microvascularization, but to a far lesser extent (27) with only a $20 \%$ increased mean intervascular distance and no effect on the individual vessel segment length. Another tyrosine kinase receptor inhibitor, ZD6474, yielded somewhat better results with a $21 \%$ increase in the intervascular distance and a $45 \%$ increase in the interbranching distance (28).

Microvessel density counts in anti-CD31 stained sections revealed $136 \%$ higher vessel surface areas in controls than in the CT-322 treated tumors. In bevacizumab treated coloadenocarcinoma line tumors (29), a microvessel reduction of only $20.1 \%$ was seen.

The VEGF-trap used in the prevention model study by Verheul et al (30) resulted in a 66\% reduction in microvascular density in hot-spots, whereby in the present study a vessel density reduction with CT-322 in an intervention model was realized not only in hot spots, but in the whole tumor.

When comparing these data it should be kept in mind that the microvessel density count assesses all endothelialized vessels irrespective of their functional status, whereas 3D microvascular corrosion casting only provides reliable data on functionally open, unobstructed vessels at that point in time when perfusion is performed.

The results presented in this study are well in line with recent findings in an orthotopic pancreatic cancer carcinoma xenograft model in which CT-322 treated tumors revealed a $76 \%$ MVD reduction (17). The impact on tumor microvasculature was paralleled by significant reductions in tumor sizes indicating that the primary cause for growth delay in this intervention model was nutrient and/or oxygen deprivation.

Typical features of many tumors are structural heterogeneities and the occurrence of hypoxic areas due to initial hypovascularization and to the malfunction of angioadaptation. Tumor blood flow may be chaotic with high flow rates in some vessel segments and stagnation in others $(31,32)$. Abnormal capillary elongations as well as sinusoidal vessels originating and draining into veins explain the low $\mathrm{pO}_{2}$ (33).

The adaptation of vessel diameter represents a potent mechanism to regulate hemodynamic properties of tumor microvasculature (32). In our study, however, no significant changes in vessel diameters and only a tendency towards more uniform vessel diameters in CT-322-treated tumors were observed.

The results presented here show a marked 'remodeling' of tumor vasculature after treatment with the Adnectin CT-322. This 'remodeling' of tumor microvasculature may improve the delivery of oxygen and drugs to tumor cells which facilitate the susceptibility of radiotherapy and the tissue penetration of therapeutics in tumors. A normalized tumor vasculature alleviates peritumoral fluid accumulation and, at the same time, decreases the shedding of tumor cells into the peripheral tissue (34). In contrast, tortuous occluded tumor microvasculature shows flow stagnation and a lack of vasculature during chemotherapy with hypoxic regions in the tumor which are inaccessible to the cytotoxic drugs (35).

These observations have profound implications in the use of anti-angiogenic therapy and for the evaluation of combinatory therapy with radio- and chemotherapy (36-38). Indeed, vascular heterogeneity may have an important influence on tumor microenvironment and its nutritive gradients.

Functional molecular tomography was recently validated as a highly sensitive method for the quantitation of tumor 
angiogenesis and therapeutic modulation $(39,40)$. Our FMT results are in striking line with the static morphometric data: in controls, FMT showed 2.5 times higher vascular volumes than those found in the CT-322 treated tumors, whereas microvascular corrosion casting showed a ratio of 1:2.42 and vascular surface areas as determined by CD31 staining of 1:2.36. The reduction on accumulation of the vascular imaging probe, AngioSense, on day 1 after a single dose of CT-322, is suggestive of reduction of tumor vascular permeability compared to vehicle-treated mice. Thus, a maximal antivascular effect of CT-322 on the tumor vasculature, mechanistically precedes and thereon sustains inhibition of tumor progression over the entire study period.

The findings of marked tumor vessel remodeling effects of CT-322 on tumor corrosion casts along with the modulation of vascular permeability function by FMT imaging, are compelling evidence that the antitumor activity of CT-322 can be mechanistically supported via a combination of suppression of VEGFR-2-mediated angiogenesis $(17,18)$ and ensuing vascular remodeling; by itself, this resulting remodeling may significantly impact sensitivity and drug delivery to partnering therapies. Considering that vascular remodeling is an important approach for cancer therapy, these results support the evaluation of the CT-322 Adnectin under combinatorial strategies and scheduling to potentially maximize the therapeutic benefit of standard of care therapies.

In conclusion, this is the first study, to our knowledge, to show an engineered novel targeted biologic with a potent antiangiogenic class effect, as well as the property to alter the chaotic tumor vascular architecture to a normalized microcirculation. Currently, CT-322 is in clinical development for the treatment of various cancers.

\section{Acknowledgements}

This work was supported by Adnexus, a Bristol-Myers Squibb R\&D Company, Waltham, MA, USA. The authors thank Dr Bickes-Kelleher for the critical review of the manuscript and Ms. Kerstin Bahr for her skillful technical assistance.

\section{References}

1. Dvorak HF, Brown LF, Detmar M and Dvorak AM: Vascular permeability factor/vascular endothelial growth factor, microvascular hyperpermeability, and angiogenesis. Am J Pathol 146: 1029-1039, 1995.

2. Hanahan D and Folkman J: Patterns and emerging mechanisms of the angiogenic switch during tumorigenesis. Cell 86: 353-364, 1996.

3. Benjamin LE, Golijanin D, Itin A, Pode D and Keshet E: Selective ablation of immature blood vessels in established human tumors follows vascular endothelial growth factor withdrawal. J Clin Invest 103: 159-165, 1999.

4. Konerding MA, Fait E, Dimitropoulou C, et al: Impact of fibroblast growth factor-2 on tumor microvascular architecture. A tridimensional morphometric study. Am J Pathol 152: 1607-1616, 1998.

5. Mancuso MR, Davis R, Norberg SM, et al: Rapid vascular regrowth in tumors after reversal of VEGF inhibition. J Clin Invest 116: 2610-2621, 2006

6. Ruoslahti E: Specialization of tumour vasculature. Nat Rev Cancer 2: 83-90, 2002.

7. Bergers $G$ and Benjamin LE: Tumorigenesis and the angiogenic switch. Nat Rev Cancer 3: 401-410, 2003.

8. Verheul HM and Pinedo HM: Possible molecular mechanisms involved in the toxicity of angiogenesis inhibition. Nat Rev Cancer 7: 475-485, 2007.
9. Jain RK: Normalization of tumor vasculature: an emerging concept in antiangiogenic therapy. Science 307: 58-62, 2005.

10. Kerbel RS: Antiangiogenic therapy: a universal chemosensitization strategy for cancer? Science 312: 1171-1175, 2006.

11. Blagosklonny MV: How Avastin potentiates chemotherapeutic drugs: action and reaction in antiangiogenic therapy. Cancer Biol Ther 4: 1307-1310, 2005.

12. Ma J and Waxman DJ: Combination of antiangiogenesis with chemotherapy for more effective cancer treatment. Mol Cancer Ther 7: 3670-3684, 2008.

13. Koide A, Bailey CW, Huang $X$ and Koide S: The fibronectin type III domain as a scaffold for novel binding proteins. J Mol Biol 284: 1141-1151, 1998.

14. Xu L, Aha P, Gu K, Kuimelis RG, Kurtz M, Lam T, et al: Directed evolution of high-affinity antibody mimics using mRNA display. Chem Biol 9: 933-942, 2002.

15. Parker MH, Chen Y, Danehy F, Dufu K, Ekstrom J, Getmanova E Gokemeijer J, Xu L and Lipovsek D: Antibody mimics based on human fibronectin type three domain engineered for thermostability and high-affinity binding to vascular endothelial growth factor receptor two. Protein Eng Des Sel 18: 435-444, 2005.

16. Getmanova EV, Chen Y, Bloom L, Gokemeijer J, Shamah S, Warikoo V, et al: Antagonists to human and mouse vascular endothelial growth factor receptor 2 generated by direct protein evolution in vitro. Chem Biol 16: 549-556, 2006.

17. Dineen SP, Sullivan LA, Beck AW, et al: The Adnectin CT-322 is a novel VEGF receptor 2 inhibitor that decreases tumor burden in an orthotopic mouse model of pancreatic cancer. BMC Cancer 8: 352, 2008.

18. Mamluk R, Carvajal I, Morse BA, Wong H, Abramowitz J, Aslanian S, Lim A-I, Gokemeijer J, Storek M, et al: Anti-tumor of CT-322 as an adnectin inhibitor of vascular endothelial growth factor receptor-2. mAbs 2: 199-208, 2010.

19. Weibel ER: Morphometry of the human lung: the state of the art after two decades. Bull Eur Physiopathol Respir 15: 999-1013, 1979.

20. Zachary I: VEGF signalling: integration and multi-tasking in endothelial cell biology. Biochem Soc Trans 31: 1171-1177, 2003.

21. Alitalo K and Carmeliet P: Molecular mechanisms of lymphangiogenesis in health and disease. Cancer Cell 1: 219-227, 2002.

22. Duff SE, Li C, Jeziorska M, et al: Vascular endothelial growth factors $\mathrm{C}$ and $\mathrm{D}$ and lymphangiogenesis in gastrointestinal tract malignancy. Br J Cancer 89: 426-430, 2003.

23. Duff SE, Jeziorska M, Kumar S, et al: Lymphatic vessel density, microvessel density and lymphangiogenic growth factor expression in colorectal cancer. Colorectal Dis 9: 793-800, 2007.

24. Liang WC, Wu X, Peale FV, et al: Cross-species vascular endothelial growth factor (VEGF)-blocking antibodies completely inhibit the growth of human tumor xenografts and measure the contribution of stromal VEGF. J Biol Chem 281: 951-961, 2006.

25. Gerber HP, Kowalski J, Sherman D, Eberhard DA and Ferrara N: Complete inhibition of rhabdomyosarcoma xenograft growth and neovascularization requires blockade of both tumor and host vascular endothelial growth factor. Cancer Res 60: 6253-6258, 2000.

26. Gerber HP and Ferrara N: Pharmacology and pharmacodynamics of bevacizumab as monotherapy or in combination with cytotoxic therapy in preclinical studies. Cancer Res 65: 671-680, 2005.

27. Drevs J, Müller-Driver R, Wittig C, et al: PTK787/ZK 222584, a specific vascular endothelial growth factor-receptor tyrosine kinase inhibitor, affects the anatomy of the tumor vascular bed and the functional vascular properties as detected by dynamic enhanced magnetic resonance imaging. Cancer Res 62: 4015-4022, 2002.

28. Drevs J, Konerding MA, Wolloscheck T, et al: The VEGF receptor tyrosine kinase inhibitor, ZD6474, inhibits angiogenesis and affects microvascular architecture within an orthotopically implanted renal cell carcinoma. Angiogenesis 7: 347-354, 2004.

29. Wildiers H, Guetens G, De Boeck G, et al: Effect of antivascular endothelial growth factor treatment on the intratumoral uptake of CPT-11. Br J Cancer 88: 1979-1986, 2003.

30. Verheul HM, Hammers H, van Erp K, et al: Vascular endothelial growth factor trap blocks tumor growth, metastasis formation, and vascular leakage in an orthotopic murine renal cell cancer model. Clin Cancer Res 13: 4201-4208, 2007. 
31. Pries AR, Cornelissen AJ, Sloot AA, et al: Structural adaptation and heterogeneity of normal and tumor microvascular networks. PLoS Comput Biol 5: e1000394, 2009

32. Jain RK: Determinants of tumor blood flow: a review. Cancer Res 48: 2641-2658, 1988.

33. Vaupel $\mathrm{P}$, Thews $\mathrm{O}$, Kelleher $\mathrm{DK}$ and Konerding $\mathrm{MA}: \mathrm{O}_{2}$ extraction is a key parameter determining the oxygenation status of malignant tumors and normal tissues. Int J Oncol 22: 795-798, 2003.

34. Jain RK, Tong RT and Munn LL: Effect of vascular normalization by antiangiogenic therapy on interstitial hypertension, peritumor edema, and lymphatic metastasis: insights from a mathematical model. Cancer Res 67: 2729-2735, 2007.

35. Duda DG, Jain RK and Willett CG: Antiangiogenics: the potential role of integrating this novel treatment modality with chemoradiation for solid cancers. J Clin Oncol 25: 4033-4042, 2007.

36. Huber PE, Bischof M, Jenne J, et al: Trimodal cancer treatment: beneficial effects of combined antiangiogenesis, radiation, and chemotherapy. Cancer Res 65: 3643-3655, 2005.
37. Dickson PV, Hammer JB, Sims TL, Fraga CH, et al: Bevacizumab-induced transient remodeling of the vasculature in neuroblastoma xenografts results in improved delivery and efficacy of systematically administered chemotherapy. Clin Cancer Res 13: 3942-3950, 2007.

38. Dings RPM, Loren M, Heun H, McNiel E, et al: Scheduling of radiation with angiogenesis inhibitors anginex and avastin improves therapeutic outcome via vessel normalization. Clin Cancer Res 13: 3395-3402, 2007.

39. Montet X, Ntziachristos V, Grimm J and Weissleder R: Tomographic fluorescence mapping of tumor targets. Cancer Res 65 : 6330-6336, 2005.

40. Montet X, Figueiredo JL, Alencar H, Ntziachristos V, Mahmood U and Weissleder R: Tomographic fluorescence imaging of tumor vascular volume in mice. Radiology 242: 751-758, 2007. 